\title{
MR imaging of penetrating spinal trauma
}

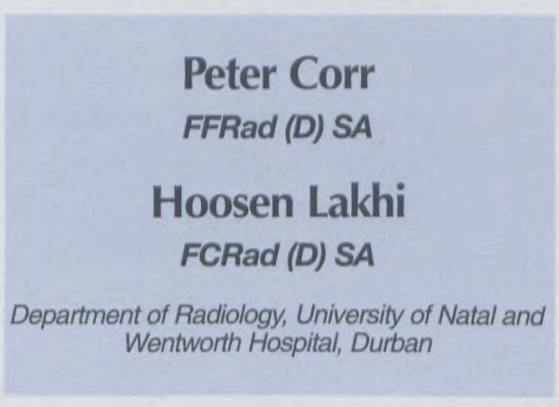

\section{Abstract}

Objective. To determine the utility of MR imaging in the assessment of spinal cord injury following penetrating spinal trauma.

Methods. We retrospectively reviewed the case notes and MR studies of patients referred to our MR facility over a 2-year period with neurological deficits following penetrating spinal injuries. All MR studies were performed on the same MR scanner with identical protocols. We reviewed the MR studies blinded to the clinical data and MR reports. We recorded the presence of: spinal cord transection, cord contusion, haematomyelia, extramedullary haematoma and vascular injury.

Results. 20 patients (17 males, 3 females) with a mean age of 28 years were studied. Causes of injury were knife wounds in 17 patients and gun shot wounds in 3 patients. Site of injury was: cervical in 14, thoracic 4 , lumbar in 2. Neurological deficits were: Brown-Séquard syndrome in 8 , paraparesis in 7 , quadraparesis in 2 , hemiplegia 2, unconscious 1 . MR findings were: partial cord transection in 9 patients, cord oedema/contusion in 5 , haematomyelia in 2 , myelomalacia in 2 , and disk herniation in 1 . One patient with cord transection sustained verterbral artery occlusion. Only 4 patients with Brown-Séquard syndrome had partial cord transection, the other 5 had cord contusions. Conclusions. MR accurately demonstrates spinal cord injury in patients with penetrating spinal trauma, however the clinical signs detected do not always correlate with the MR findings.

\section{Introduction}

Penetrating spinal trauma is the third most common cause of spinal cord injury in the USA after motor vehicle accidents and falls from a height. ${ }^{1}$ In a large series in South Africa penetrating trauma was the second most common spinal cord injury following motor vehicle accidents. ${ }^{2}$ Radiologists play an important role in the diagnosis of spinal cord injury and in predicting recovery of function following blunt spinal trauma. ${ }^{3}$ However as far as we are aware, there has been no series describing the MR findings of penetrating spinal trauma in the radiology literature. We reviewed our experience in MR imaging of patients who had sustained penetrating injuries of the spine.

\section{Patients and methods}

We retrospectively reviewed the case notes of patients who were referred for MR imaging for penetrating trauma over a 24 -month period. We recorded patient demographics, type of injury and neurological deficit.

We independently reviewed the MR studies of the patients. Both readers were experienced neuroradiologists. All studies were performed on the same 1.5T MR scanner with a dedicated spine phased array coil. All patients were imaged with the same protocol: sagittal fast spin echo T2weighted sequence $(4000 / 120$ ETL 12), sagittal fast spin echo T1weighted sequence $(500 / 30)$, gradient echo axial sequence (600/25/25 degrees). If spinal cord injury was detected a coronal T1-weighted sequence was attempted. MR angiograms were routinely performed using a $2 \mathrm{D}$ time of flight sequence.

We recorded the presence or absence of spinal cord injury, spinal cord contusion, haematomyelia and extramedullary haematoma from the MR images. Spinal cord transection was diagnosed by demonstrating the knife tract or bullet tract within the cord. Transection was demonstated by a focal T1 hypointense and T2 hyperintense lesion within the spinal cord. Spinal cord contusion was diagnosed by the presence of cord swelling with diffuse $\mathrm{T} 1$ hypointensity and $\mathrm{T} 2$ hyperintensity. Haematomyelia was diagnosed by the demonstration of blood products within the spinal cord either as $\mathrm{T} 1$ and $\mathrm{T} 2$ hyperintensities (methemoglobin) in the subacute phase between 1 and 3 weeks post injury or T1 and T2 hypointensities (deoxyhaemoglobin or haemosiderin) 
in the acute phase less than 1week after injury.

\section{Results}

We detected 20 patients from the retrospective review. There were 17 male and 3 female patients, the mean age was 28 years old ( $16-42$ years). Patients were imaged within 1 week of the injury except for 2 patients with myelomalacia imaged at 4 and 5 weeks respectively after injury. The cause of injury, neurological signs, and imaging findings are recorded in Table I. The cause of injury was knife wounds in 17 patients and gun shot wounds in 3 . The site of injury was the cervical spine in 14 patients, thoracic spine in 4 patients, and lumbar spine in 2 patients. Partial spinal cord transection was detected in 8 patients, 4 of whom had signs of the BrownSéquard syndrome (Figs 1a, b, c and Figs $2 \mathrm{a}, \mathrm{b})$. Of the other 4 patients with Brown-Séquard syndrome, 3 patients had spinal cord contusion and 1 had myelomalacia (Figs $3 \mathrm{a}, \mathrm{b}$, Figs 4a, b and Fig. 5). Vertebral artery occlusion was detected on MR angiography and confirmed on selec- tive vertebral angiography in 1 patient. Myelomalacia was detected in the cervical cord of 2 patients who presented 4 and 5 weeks after the injury (Figs $4 \mathrm{a}$ b). Only 1 patient had an extradural cervical haematoma and 2 patients sustained intramedullary haematomas.

\section{Discussion}

Patients with penetrating knife injuries commonly present with a Brown-Séquard syndrome; there is usually ipsilateral motor weakness and sensory loss for proprioception

Table I. Cause of injury, clinical signs, and imaging findings

\begin{tabular}{|c|c|c|c|c|}
\hline Patient & Cause of injury & Signs & Site & MR findings \\
\hline 1 & Knife & Brown-Séquard & Cervical & Contusion \\
\hline 2 & Knife & Paraparesis & Lumbar & Conus contusion \\
\hline 3 & Knife & Paraparesis & Thoracic & Cord transection \\
\hline 4. & Knife & Paraparesis & Cervical/T & Cord contusion \\
\hline 5 & Knife & Unconscious & Cervical & $\begin{array}{l}\text { Partial transection } \\
\text { Occipital pole } \\
\text { infarcts }\end{array}$ \\
\hline 6 & Knife & Quadraparesis & Cervical & Myelomalacia \\
\hline 7 & Knife & Brown-Séquard & Cervical & Myelomalacia \\
\hline 8 & Knife & Brown-Séquard & Cervical & Conus contusion \\
\hline 9 & GSW & Quadraparesis & Cervical & Cord haematoma \\
\hline 10 & Knife & Brown-Séquard & Thoracic & Partial transection \\
\hline 11 & Knife & Hemiplegic & Cervical & $\begin{array}{l}\text { Partial transection } \\
\text { Vertebral A } \\
\text { occlusion }\end{array}$ \\
\hline 12 & GSW & Paraparesis & Lumbar & Disc herniation \\
\hline 13 & Knife & Paraparesis & Cervical & Cord haematoma \\
\hline 14 & Knife & Brown-Séquard & Cervical & Cord contusion \\
\hline 15 & Knife & Brown-Séquard & Cervical & $\begin{array}{l}\text { Partial transection } \\
\text { Cord haematoma } \\
\text { Extradural } \\
\text { haematoma }\end{array}$ \\
\hline 16 & Knife & Brown-Séquard & Cervical/T & $\begin{array}{l}\text { Partial transection } \\
\text { Cord haematoma }\end{array}$ \\
\hline 17 & Knife & Paraparesis & Thoracic:/L & Partial transection \\
\hline 18 & Knife & Paraparesis & Cervical/T & Transected cord \\
\hline 19 & Knife & Brown-Séquard & Cervical & Partial transection \\
\hline 20 & GSW & Hemiplegia & Cervical/T & Cord contusion \\
\hline
\end{tabular}




\section{ORIGINAL ARTICLE}
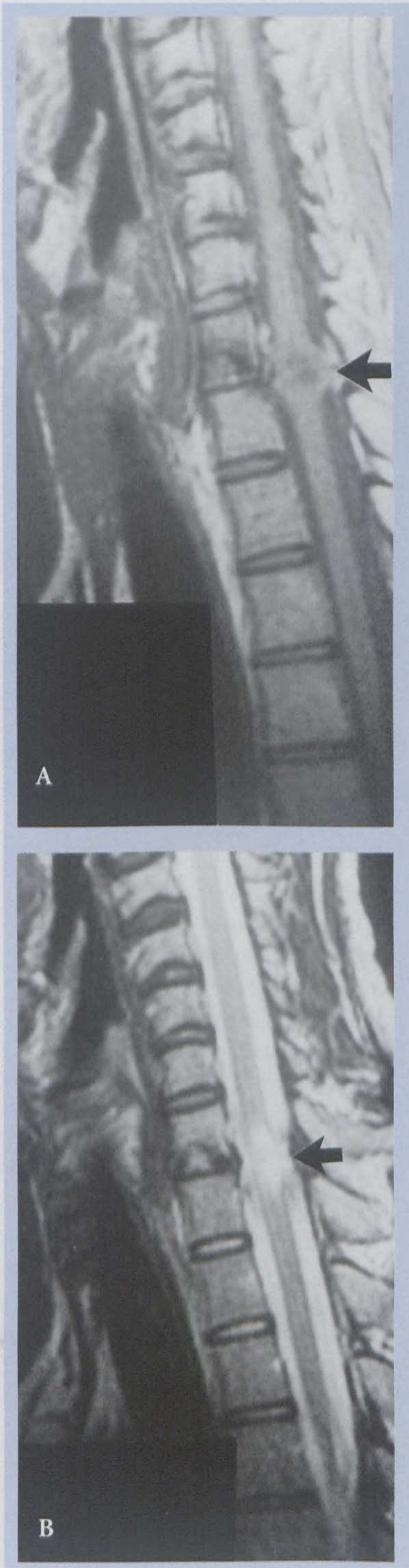

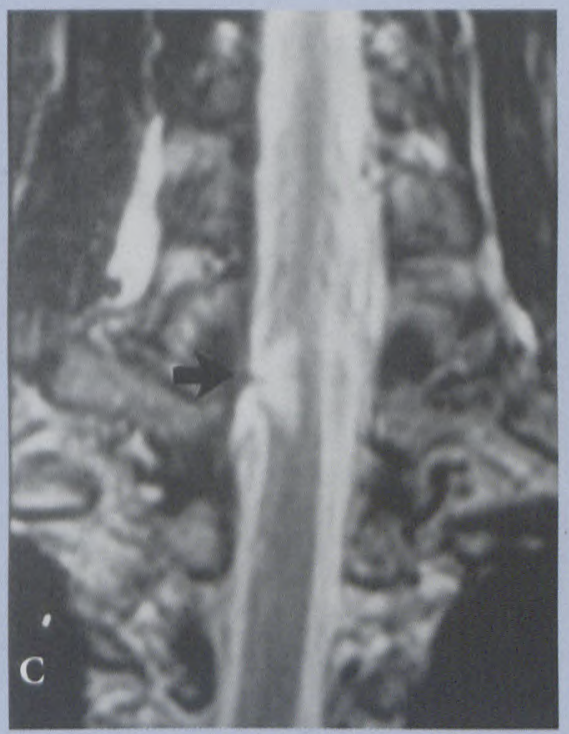

Fig. 1a, b, c. T1 and T2 sagittal and coronal MR scans of a patient (2047/98) who presented with Brown-Séquard syndrome demonstrating partial cord transection at the cervicothoracic junction following a knife wound (arrows).

and contralateral sensory loss to pain and temperature below the level of injury. On MR imaging these patients may have evidence of a partial transection of the spinal cord in an oblique or parasagittal plane or focal cord contusion. It is important to realise that Brown-Séquard syndrome may also be due to focal cord contusion which often resolves, while patients with partial cord transection do not usually recover function. This has been detected in patients with Brown Séquard syndrome following blunt cervical trauma. ${ }^{4}$

The knife tract appears as a focal hypointense lesion on T1-weighted images and hyperintense on T2weighted images, unless there is haematoma along the tract in which case the tract will appear hyperintense on T1 and T2-weighted images in the subacute phase between 1 and 2 weeks post injury. We found coronal imaging particularly useful in these patients to assess the extent of the partial transection (Fig. 1c). Knife injuries usually follow dorsal stab wounds
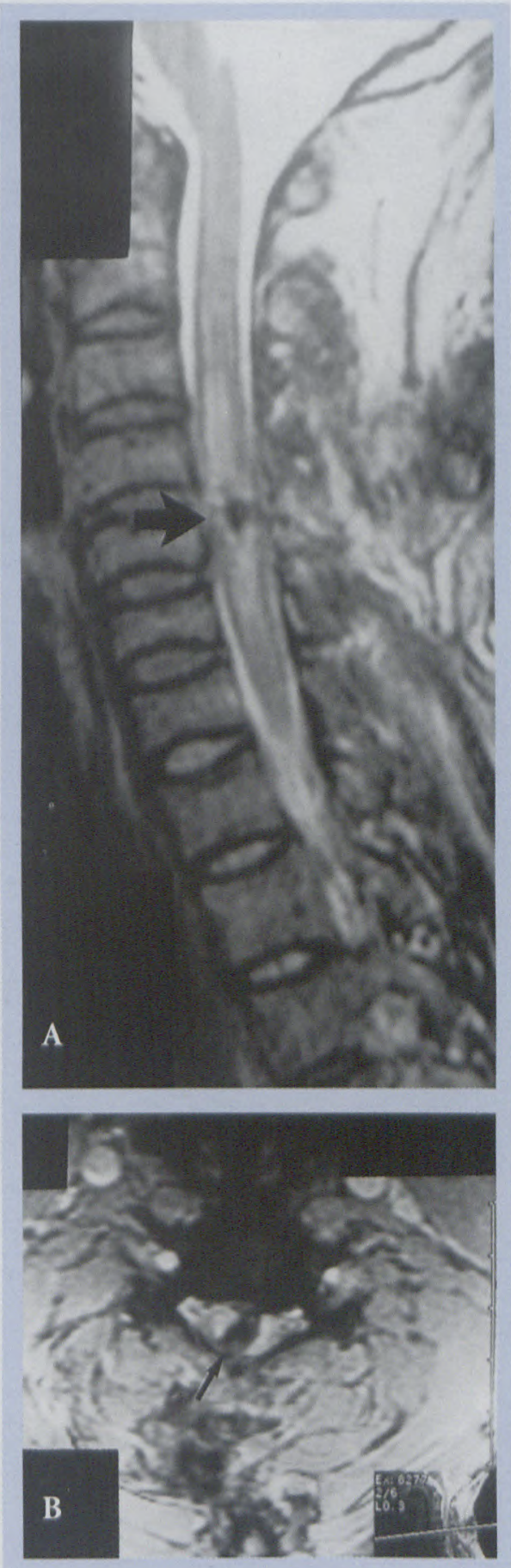

Fig. 2a, b. T2 sagittal and gradient recalled axial MR of a patient who sustained a knife injury to the cervical spine 2 weeks prior to the study. MR demonstrates knife tract, haematomyelia (haemosiderin staining) and hemisection of the cord (arrows).

with the knife tip entering the spinal canal between the laminae in the gutter formed by the spinous process and the transverse process. ${ }^{5}$ This results in 


\section{ORIGINAL ARTICLE}
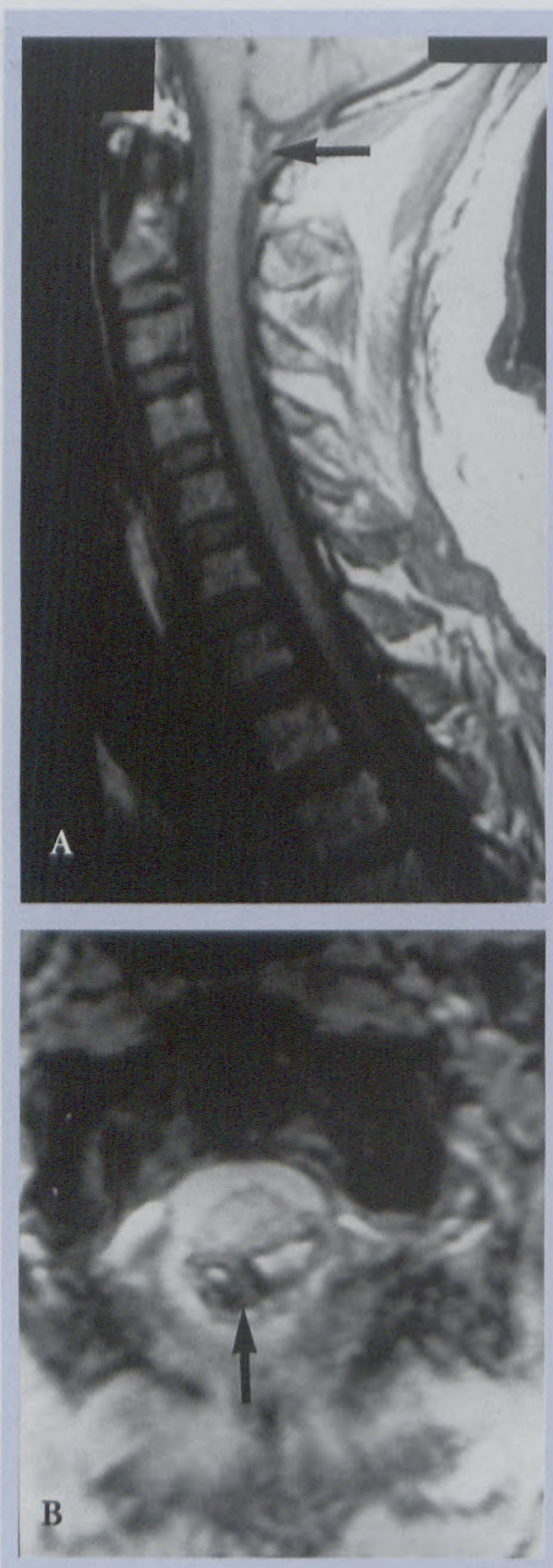

Fig. 3a, b. MR T1 sagittal and gradient recalled axial of a patient with quadraparesis following a C1 knife injury demonstrates posterior intra and extradural haematoma and cord contusion (arrows).

hemitransection of the cord.

Patients with Brown Séquard syndrome following spinal cord contusion usually recover function, unlike patients with partial transection or intramedullary haematoma. ${ }^{4,5}$ Often the injury was on the opposite side of the cord to the initial site of the stab wound. Peacock et al. ${ }^{5}$ postulated that
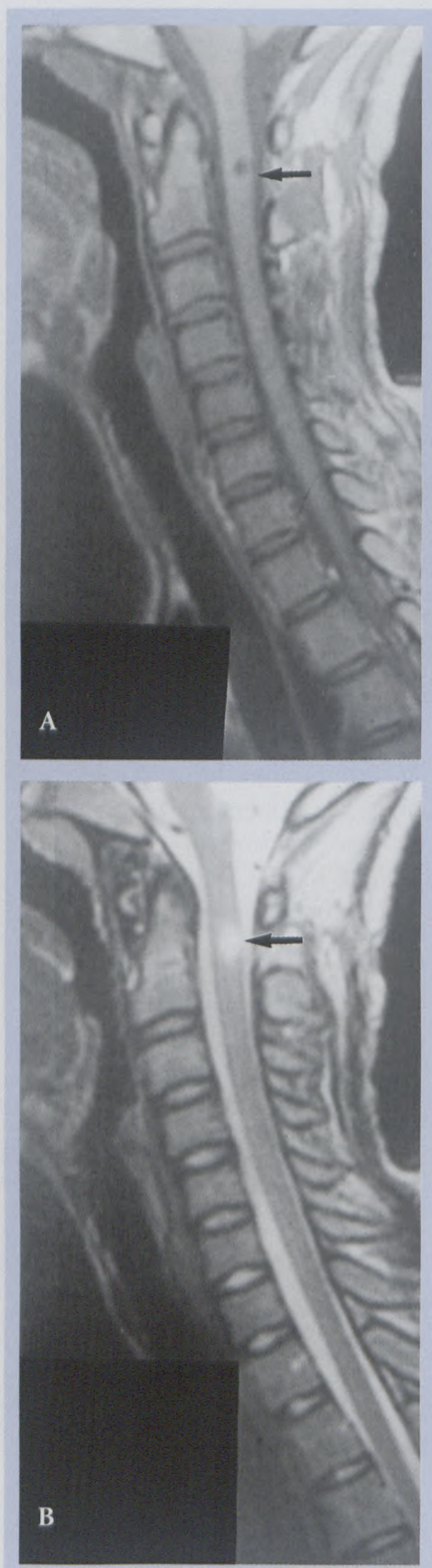

Fig. 4a, b. MR T1 and T2 sagittal scans of a patient with quadraparesis following a stab injury 5 weeks previously demonstrates focal myelomalacia at the C2 level (arrows).

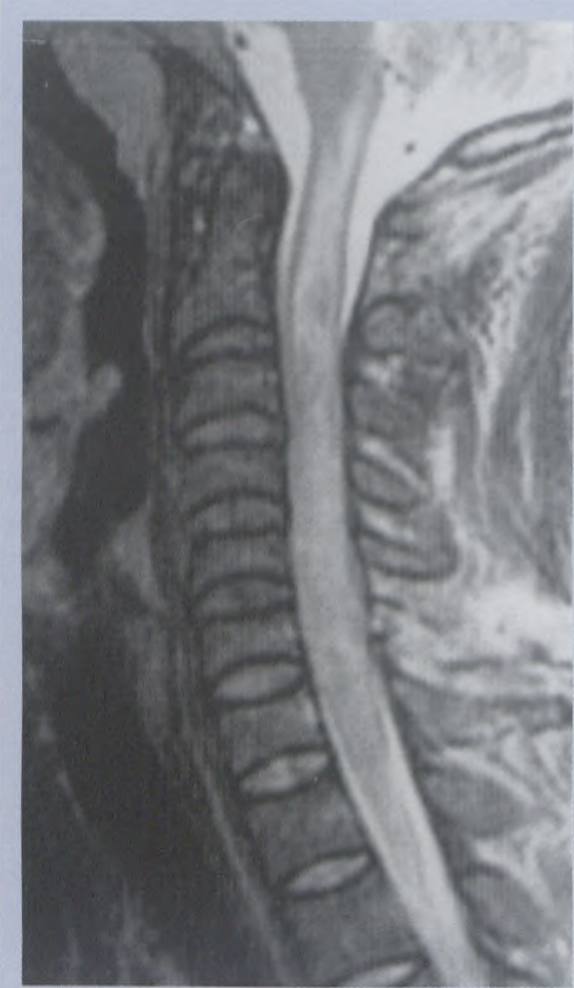

Fig. 5. T2 sagittal scan of a patient who developed quadraparesis following a penetrating gun shot wound to the cervical spine demonstrates extensive spinal cord contusion.

these patients sustained a contrecoup contusion of the cord as the cord was compressed against the bony canal by the knife blade. In Peacock and coworkers' series of 450 spinal cord stab injuries, of which one-half were the Brown Séquard syndrome, they found that two-thirds of patients made a functional recovery. ${ }^{5}$ This study was performed before MR was available and it is likely that many patients actually had spinal cord contusions rather than partial transections. The presence of extramedullary haematoma was uncommon in our series and did not appear to influence the outcome. Vertebral artery occlusion was established in one patient on MR angiography and selective vertebral angiography and postulated in the patient with vertebrobasilar infarction. It is likely that vertebral artery injury remains undetected in these patients unless 
specifically looked for with MR angiography. The plain radiographs were all reported to have no evidence of fractures although paravertebral and prevertebral haematomas were often detected. Although few patients had CT of the spine performed in this study, we believe that $\mathrm{CT}$ will be more accurate in detecting any bony injury to the laminae. We only had 3 patients with bullet wound injuries to the spine. Two of these patients had extensive cord contusions and 1 patient developed haematomyelia. The extensive injury to the cord is probably due to the high velocity nature of the weapons used. Metallic artefacts from bullet shrapnel may degrade the scan quality especially when gradient echo sequences are used to detect blood products. It is extremely important to be aware of the possibility of ferromagnetic shrapnel fragments within the cord or contiguous to the cord moving during the scanning process and so increasing the risk of further cord damage.

\section{Conclusion}

In conclusion, we believe that MR imaging is useful to establish the presence and nature of spinal cord injury following penetrating spinal trauma. We found that the patient's neurological signs often did not correlate with the MR findings.

\section{References}

1. Jallo GI. Neurosurgical management of penetrating spinal injury. Surg Neurol 1997; 47: 328 330.

2. Velmahos GC, Degiannis E, Hart K, Souter I, Saadia R. Changing profiles in spinal cord injuries and risk factors influencing recovery after penetrating injuries. I Trauma 1995; 38: 334-337.

3. Flanders A, Spettell C, Tartaglino L, Friedman D, Herbison G. Forecasting motor recovery after cervical cord injury: value of MR imaging. Radiology 1996; 201: 649-655.

4. Oller DW, Boone S. Blunt cervical spine Brown Séquard injuries: a report of three cases. Am Surg 1991; 57: 361-365.

5. Peacock W, Shrosbree RD, Key AG. A review of 450 stabwounds of the spinal cord. S Afr Med J 1977; 51: 961-964.

\section{FOR SALE \\ HOLOGIC QDR 4500 ELITE DEXA SCANNER}

- On maintenance contract with Scientific Group

- Excellent condition, guaranteed by Scientific Group (sole importers of Hologic machines)

- Transportation and installation paid for by us

For further inquiries or to view please contact Leigh-Anne (011) 884-2162

Block A Rochester Place, 173 Rivonia Road, Morningside, Sandton

\section{Professor of Radiology}

Discipline of Radiology

Faculty of Medical and Health Sciences

\section{Vacancy A220-03}

The University of Auckland Faculty of Medical and Health Sciences is seeking applications for the established Chair in Radiology which is a joint University/Hospital appointment with university conditions and specialist status within the teaching hospitals of Auckland Healthcare.

Candidates must hold a recognised postgraduate qualification and be eligible for vocational registration by the Medical Council of New Zealand. The successful candidate will be expected to provide research leadership in the imaging sciences, teach at undergraduate and postgraduate levels and spend half his/her time in clinical activities. Prior experience in research and teaching is expected. Limited private practice is permitted.

Enquiries of an academic nature should be directed to Associate Professor Cynthia Jensen, Head of Department of Anatomy with Radiology, telephone 64-9-373 7599 ext.86056; Fax 64-9-373 7484; email: c.jensen@auckland.ac.nz.

For further information and to apply on-line, please visit

www.vacancies.auckland.ac.nz or alternatively call 64-9-373 7599 ext 83000. Please quote the vacancy number.

\section{Applications close on 29 August 2003.}

The University has an EEO policy and welcomes applications from all qualified persons. 